\title{
A Formal Description of the Systemic Theory based e-Learning
}

\author{
Gabriela Moise
}

\begin{abstract}
This paper aims at presenting the systems theory-based approach to the learning (instructional) process. Such as approach is necessary, if one takes into consideration the the complex and holistic nature of the learning process. The presented modeling is based on the definition of an automatic regulation system and artificial intelligence techniques. For this purpose, an Intelligent Instructional System (IIS) is defined using the concepts: inputs, outputs, perturbations factors, regulation, feedback and AI learning techniques. The contextual environment of learning plays the major role in this system and it establishes the perturbation factors. The regulation consists in AI learning techniques, so the IIS is a flexible and adaptive system. The process' behaviour is described using pseudo-boolean and linear equations. This kind of approach enables solving the problems of the complexity and uncertainty of the learning (instructional) systems and, particularly, those of the e-learning (online and offline). Applications of the proposed approach may be found in e-learning courses for Mathematics, Computing, Architecture, Archaeology, Cultural Heritage, etc.
\end{abstract}

Keywords: online learning, system theory, feedback, intelligent instructional system.

\section{Introduction}

The theoretical bases of the instructional process design are formed of a set of theories such as the instruction and learning theory, the communication theory, the systems' theory. The system theory and the systemic thinking have a major influence on the field of the instructional design. The systemic approach of the instruction process design was issued by James Finn [7], who applied the systems theory in the educational technology. In 1978, Walter Dick and Lou Carey published the book entitled "The Systemic Design of Instruction". The model built by Dick and Carey [5] doesn't follow a linear approach, each level of the instructional process being revised for certainty of achieving the objectives of instructional process. The model is presented in figure no. 1.

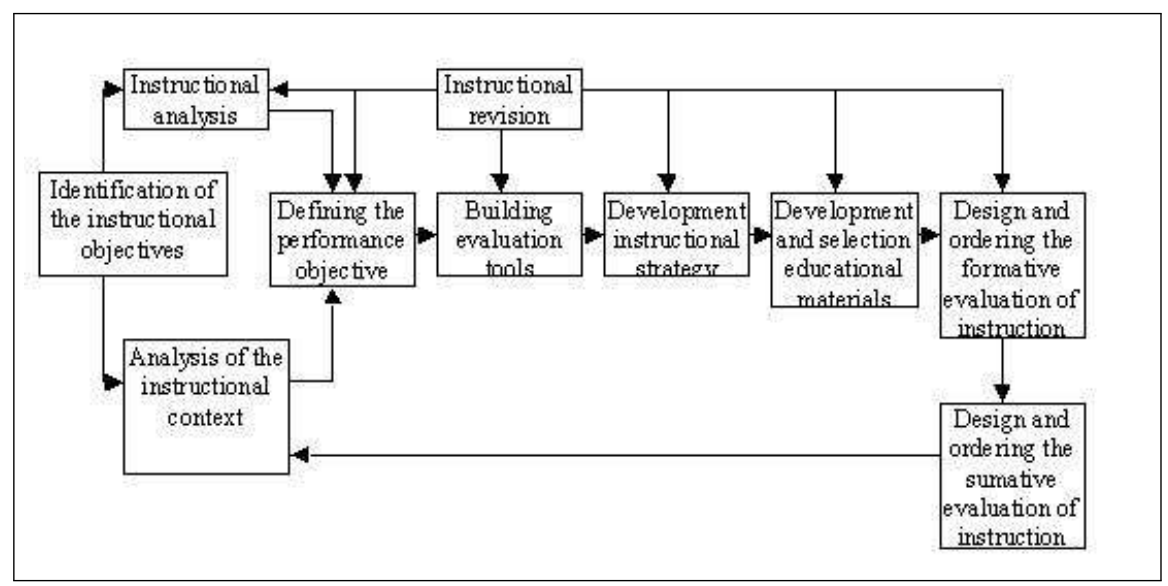

Figure 1: Dick and Carey's model

Any learning (instructional) form may be tackled from the point of view of the general systems theory, distance learning forms included. A system is defined by a set of elements that interact and work together in order to achieve an objective. Moore and Kearsley [15] present the importance of the 
systemic approach of the distance educational process. The distance educational system has to be viewed as a system within which all the elements are interdependent and interconnected. The consequence of this fact is that any change of an element of the system will affect the whole instructional process.

Moore (1983) defined the concept of transactional distance, in that he rigorously presented the relation between the teacher and the student: the distance between teacher and student is a geographical, an educational and a psychological one.[16]

Saba [17] refined the theory of Moore and proved trough experiments, that the transactional distance is a measure of the relation existing between the teacher and the student. Saba asserts: "transactional distance varies by rate of dialogue and structure", so that when dialogue increases, the structure and transactional distance decrease; when the structure increases, the transactional distance increases and the dialogue decreases (see figure 2).

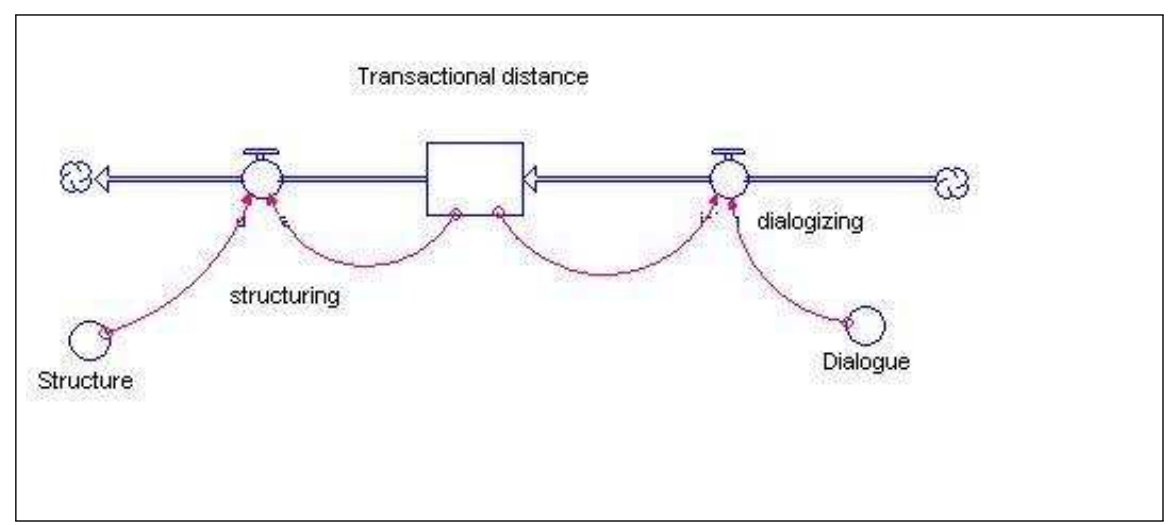

Figure 2: STELLA modeling of dynamic of Saba's system

transactional distance $(t)=$ transactional distance $(t-d t)+($ dialogue - structure $) * d t$

Norbert Wiener [20] has introduced the cybernetic concept in the paper "The Human Use of Human Beings": "when we desire a motion to follow a given pattern the difference between this pattern and the actually performed motion is used as a new input to cause the part regulated to move in such a way as to bring its motion closer to that given by the pattern."

The notion of instructional system was introduced by Robert Glaser in 1962. The levels of the system defined by Glaser are represented in the figure no. 3. The individualized instruction is viewed as a continuous cycle of diagnose, specification and evaluation. The output condition is defined as follows: the student has learned all the proposed objectives.

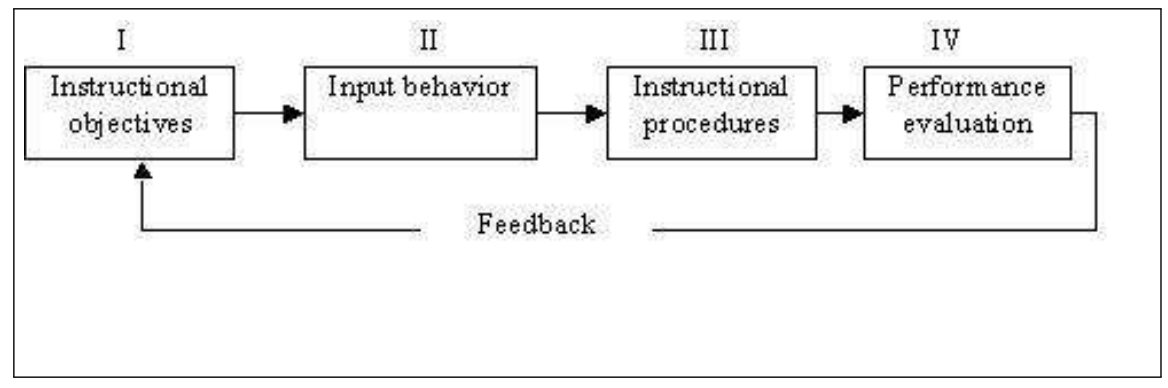

Figure 3: Glaser's instructional model with feedback

Banathy (1996) [2] comprised the educational system in the human activity systems (HAS). He defined the term of "system view of education": "we learn to think about education as a system, we can understand and describe it as a system, we can put the systems view into practice and apply it in educational inquiry, and we can design education so that it will manifest systemic behavior". The 
systemic approach of the educational system facilitates the exploration of the process according to the subsystems constituents: teacher, student, institution, administration etc. Banathy (1992) [1] proposed two phases of the systemic development of education, which are considered the principles of systems' design. (see figure 4)

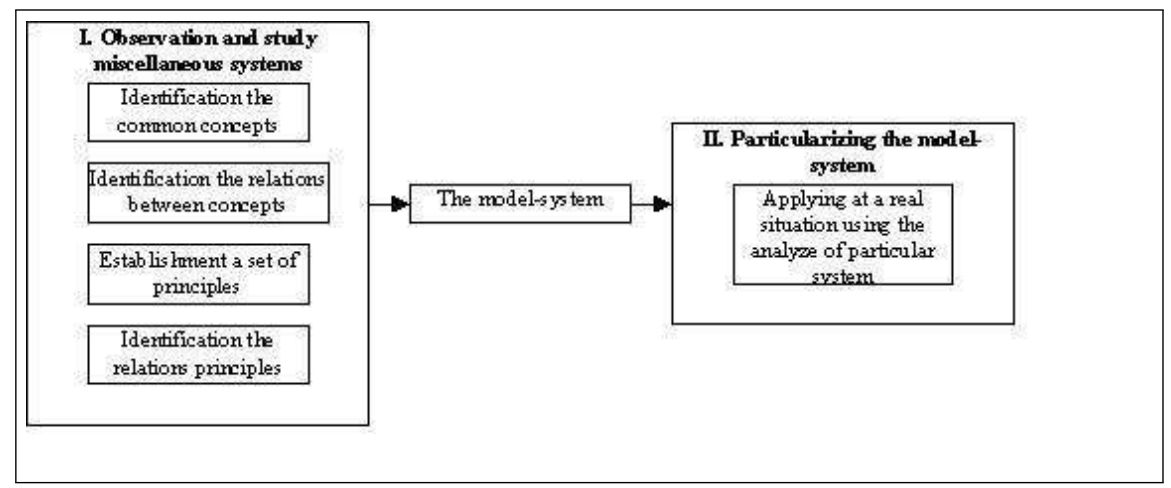

Figure 4: The phases of the development of a systemic approach of education (Banathy)

In 1992, Banathy [1] proposed three models of social systems namely the systems-environmental model, the functions-structure model and the process-behaviour model. These models were be used to describe the educational systems "open, dynamic and complex social systems". The systems-environmental model enables one to see the learning (instructional) systems into the context of the relationships, interactions and interdependencies between the system and the environment. The functions-structure model enables one to describe the goals of the systems and the functions that have to be implemented in order to achieve these goals. Also it defines the components of the system and the relations between them, thus defining the very structure of the system. The process-behavior model enables one to understand the behaviour of the system: which are the inputs of the system, how these inputs have transformed to produce the expected output. Also, Banathy identified four domains: systems' philosophy, theory, methodology, and application, constituting the conceptual system of systems inquiry in educational systems. A detailed report of the systems view of the distance learning may be found in [6]. The e-courses have to be design to incorporate active and cooperative learning techniques in a systematic way that addresses all aspects of the course: delivery, management, and assessment.[9]

In this paper, the author proposes a new model of the learning (instructional) process based on the systems theory. The model focuses on the regulation, so that the system will produce the expected results. The behaviour of the system is described using a mathematical model, based on the pseudoboolean equations and linear equations, which was called "quasi-boolean system". The concept of the "quasi-boolean system" is introduced to portray the behaviour of the e-learning system. The genesis of the "quasi-boolean system" is the idea that the students are evaluated at the end of a training session and they get a grade: a number or a truth value (true or false). In the traditional learning (instructional) process, the teacher takes care of achieving the objectives of the learning (instructional) process. In the e-learning system, one uses a combined regulation: a feedback regulator and a predictive regulator. The predictive regulator uses AI learning techniques (Q-learning [13]).

The remaining part of the paper is organized as follows: in section 2, a systematic model of the learning (instructional) process is presented; in Section 3, a mathematical model is given; in section 4 , an application is developed; in section 5 the complexity of the instructional (learning) process is identified and finally, in section 6 Summary and Conclusions, the advantages using the proposed model are presented. 


\section{A Systemic Model of the Learning (Instructional) Process}

The multidisciplinary nature of the systems theory facilities the implementation of the system thinking in the learning (instructional) process.[3]

In this paper, the learning (instructional) process is modeled starting from the definition of an automatic regulation system.

Definition 1. An automatic regulation system (S,R,A) is defined according to the schema presented in figure no. 5.[11]

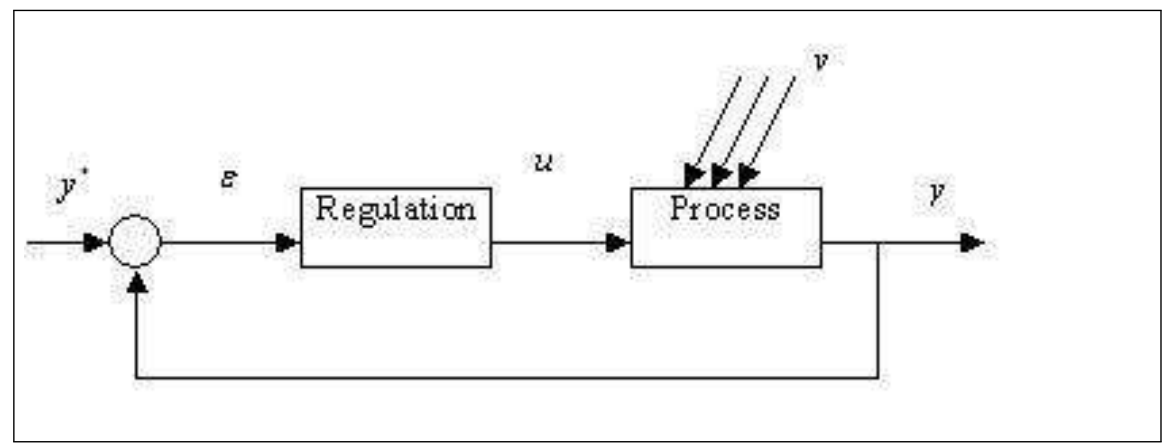

Figure 5: An automatic regulation system

$y^{*}$ is the reference value, $y$ is the output value, $v$ are the perturbation factors, $u$ is the input value, $\varepsilon$ is the error between the reference value and the output value.

Definition 2. An intelligent instructional system (IIS) with a feedback regulator is defined according to the schema:

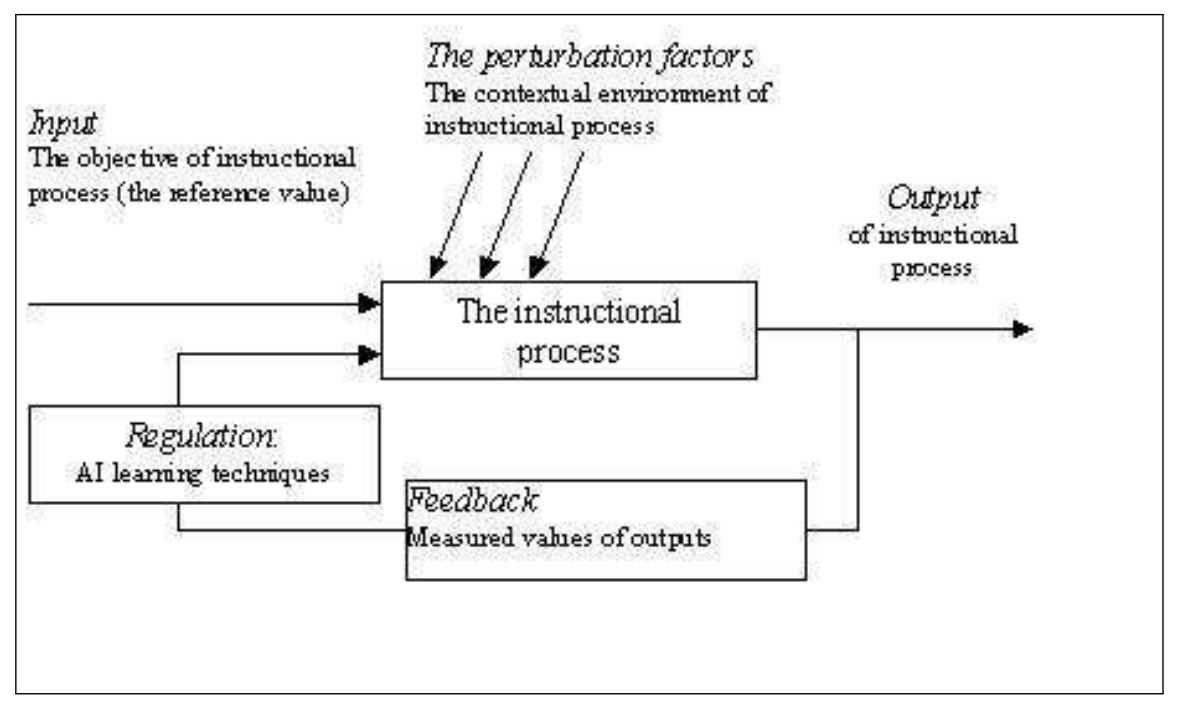

Figure 6: The intelligent instructional system

The instructional system is a system with inverse connection that decides the behavior view to nullify the error.

Definition 3. An intelligent instructional system (IIS) with a combined regulation is defined according to the schema presented in the figure no. 7. 


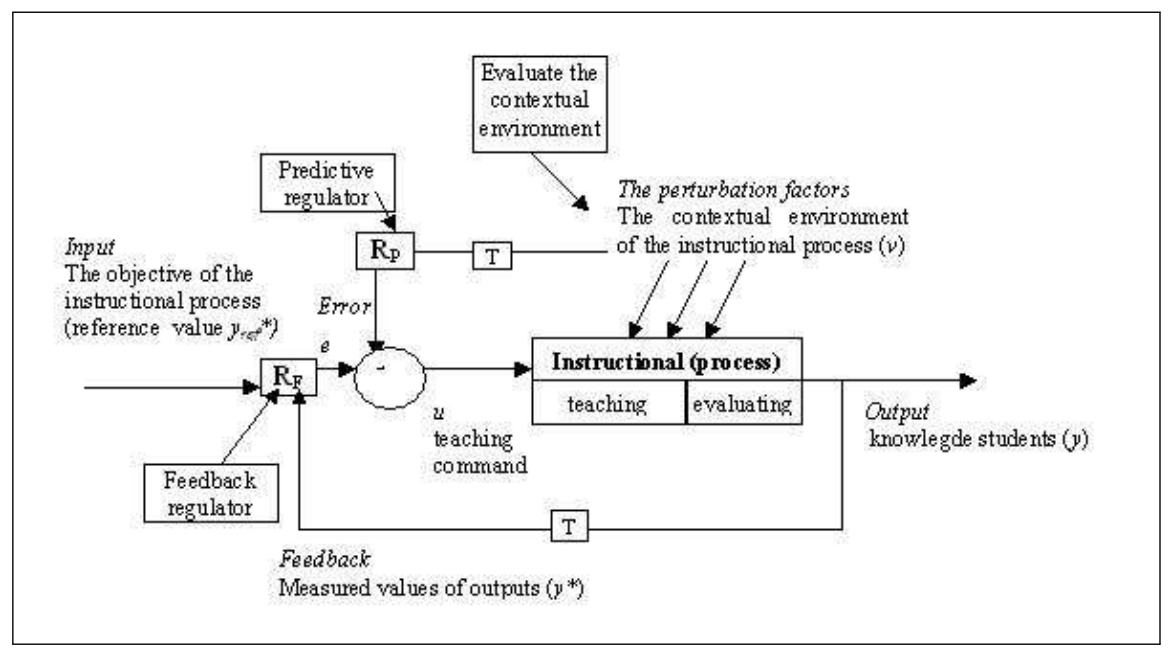

Figure 7: Intelligent instructional system

The input of the system represents the reference value, what the students have to realize after the learning (instructional) process has finished. The state at $k$ step (the system is sequential) is defined by the students' knowledge and skills at step $k$. The instruction context may be referred as a social, an emotional, a mental, school, technological, knowledge context.[14] In this approach, all factors that affect the learning - motivation, goals, previous knowledge, interest, teaching styles, learning styles, classroom climate, parents, preoccupations, hobbies, etc - represent the perturbations.

Definition 4. An intentional model of instruction is a structure with the form: (com,con,cri), where com, con, cri have the following meanings:

- com defines the behaviour

- con describes the conditions in which the students prove learning

- $c r i$ is a precise setting of acceptable standards and performance

The teacher has to consider the inferences in the learning process, starting from measurable pieces of evidence that can be measured, while the learning can not be measured directly. The goals of learning are formulated according to the domains of learning: the cognitive domain, the affective domain and the psychomotor one. The teacher and the instructional designer have the major role in defining the goals of learning.

Definition 5. The input values of IIS are defined by the goals of the instructional process, the intentional models of behaviour.

The goals of a course describe the intention of the teacher referring to the students, who attend the course. The goals define what is expected from the students after the instructional process has finished.

$y_{\text {ref }}^{*}=\left(\operatorname{com}_{r e f}^{*}, \operatorname{con}_{r e f}^{*}\right.$, cri $_{r e f}^{*}$, grade $\left._{r e f}\right)$, where

- $\operatorname{com}_{\text {ref }}^{*}$ defines the reference behaviour

- $\operatorname{con}_{r e f}^{*}$ describes the reference conditions in which the students prove learning

- $c r i_{r e f}^{*}$ is the setting of the reference standards and performance

- $\operatorname{grade}_{r e f}^{*}$ is the reference grade (the accepted grade) 
For example:

$y_{\text {ref }}^{*}=$

(behaviour: write aprogram, conditions: using $C++$ language in 15 minutes,

criteria : program runs without errorsand displaycorrect results, 8 )

or

$y_{\text {ref }}^{*}$

$=($ write a program,$C++$ language, 15 minutes, programs run without errors, displaycorrect results, 10$)$

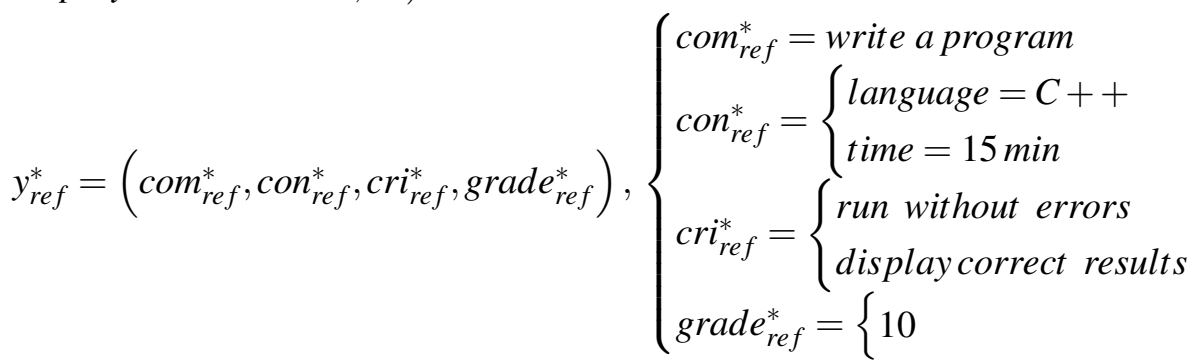

Meaning: At the end of the module, the student will be able to write a program to sum the first $n$ integer numbers, using $\mathrm{C}++$ language, in 15 minutes, the program will run without errors and the result will be correct, the reference grade is 10 .

Definition 6. The outputs of the IIS system are defined as follows: $y=\left(y_{1}, y_{2}, \ldots, y_{m}\right)$, where $y_{i}$ are defined according to Gagne [8]

- Declarative knowledge;

- Procedural knowledge;

- Cognitive strategies;

- Attitudes;

- Psychomotor abilities.

The outputs (the students' knowledge) are measured $\left(y^{*}\right)$ in a way that allows them to be compared with the reference values.

In order to evaluate the outputs of the IIS system, a measuring system (MS) is to be established.

$M S: Y \rightarrow M, \mathrm{Y}$ is the possible outputs' sets.

The measuring system has to take into account goals of the instruction (the reference value of the IIS system). The behaviour of the student has to be evaluated in the conditions defined by the goals of the instruction and has to satisfy a set of criteria. The measuring system evaluates the outputs of the system (what the student realize in the evaluation phase) and produces feedback, so the measuring system establishes an output behaviour model.

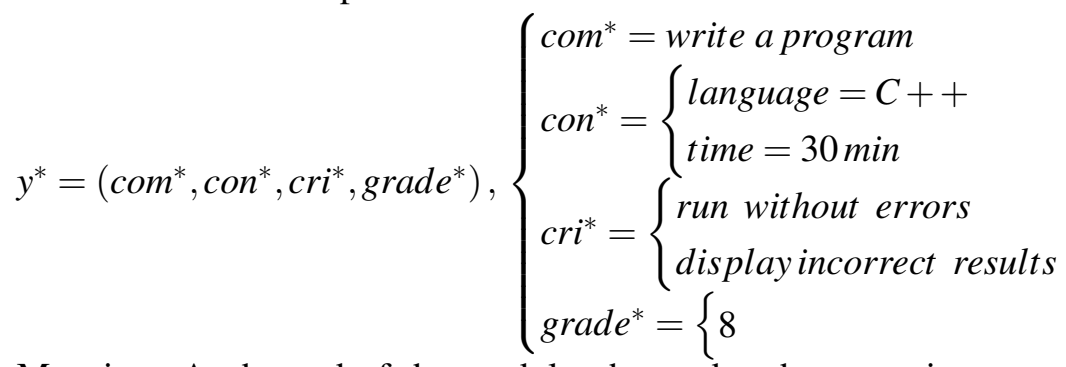

Meaning: At the end of the module, the student has to write a program to sum the first $\mathrm{n}$ integer numbers, using $\mathrm{C}++$ language, in 30 minutes, the program runs without errors and the result is correct, the grade is 8 . 
The output of the IIS system is evaluated according to the MS and has the following values:

$y^{*}=M S(y)=($ com, con, cri, grade $)$

The error is defined as a difference between two models (patterns). To determine the difference between two behaviour patterns, a patterns' analyzer (defined through a procedures collection) will be used.(see figure 8)

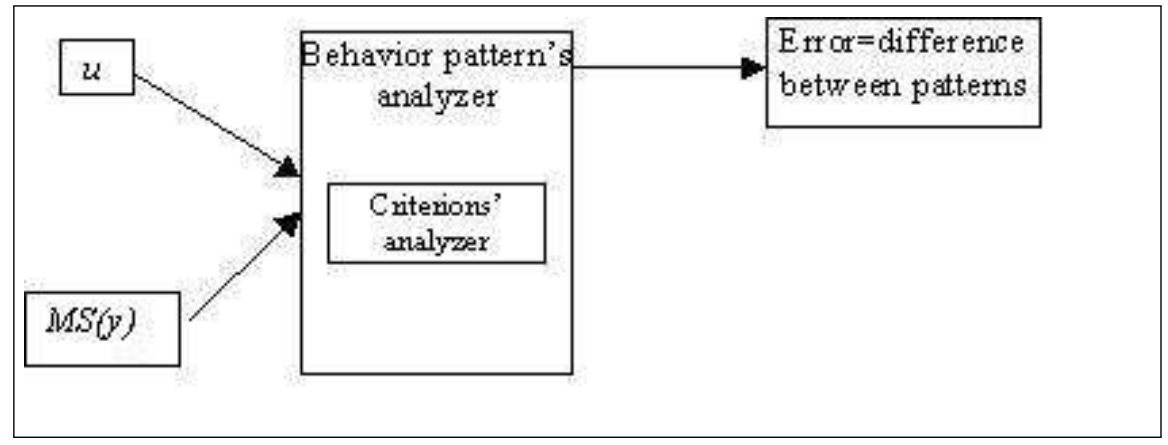

Figure 8: Patterns' analyzer

The analysis of the behaviors' patterns imply the following steps:

1. the criteria will be decomposed in primitive criteria;

2. the primitive criteria are analyzed according to some rules based procedures;

3. the analysis procedures are updated according to learning techniques taken from artificial intelligence.

In the example above:

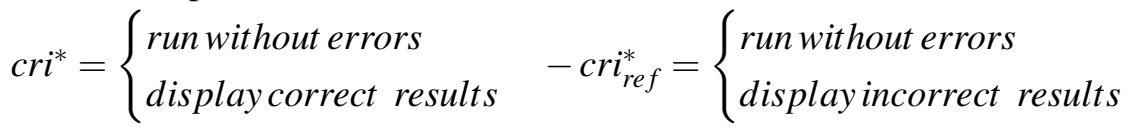

The criteria analysis establishes that the program written by the student has a logical error. The value of the error: invalid logical program.

Definition 7. The perturbation factors are defined by the contextual environment of the learning (instructional) process.

The contextual learning is built on all conditions that affect the instructional process. Cole and Griffin have shown the complexity of this concept.[4]

The predictive regulator of the IIS system decides what kind of instructional techniques and information will be used in the learning process, analyzing the contextual factors. The predictive regulation contains AI techniques to instruction of the IIS system. The predictive commands are rules having the following structure:

$r=\left(c_{1} \wedge c_{2} \wedge \ldots \wedge c_{n}\right) \rightarrow\left(a_{1} ; a_{2} ; \ldots ; a_{k}\right)$, where $c_{i}, i=1, \ldots, n$ are the conditions and

$a_{i}, i=1, \ldots, k$ are the actions

In the IIS system, the conditions are perturbation factors and they are inputs of the system. The feedback regulator of the IIS system checks the value of the error and provides a corrective command. While the IIS system is not a numeric system, the behaviour of the system cannot be described using equations with real numbers. 


\section{A Mathematical Model of the Instructional System}

Definition 8. A vector of $n$ elements, where the elements are real numbers and logical values, is called a quasi-boolean vector.

$x=\left(x^{1}, x^{2}, \ldots, x^{k}, x^{k+1}, \ldots, x^{n}\right)$ where $x^{i} \in \mathbf{R}, i=\overline{1, k}$ and $x^{i} \in \mathbf{B}_{2}, i=\overline{k+1, n}$

The real part of $x$ is noted with $R(x)$ and the boolean part of $x$ is noted $B(x)$ and they are defined as:

$R(x)=\left(x^{1}, x^{2}, \ldots, x^{k}\right)$

$B(x)=\left(x^{k+1}, x^{k+2}, \ldots, x^{n}\right)$

Definition 9. It is called a quasi-boolean sequential system a pair of functions $\left(f=\left(f_{1}, f_{2}, f_{3}\right), g\right)$ defined as:

$x_{k}^{i}=f_{1}^{i}\left(B\left(x_{k-1}\right), B\left(u_{k-1}\right), B\left(v_{k-1}\right)\right)+f_{2}^{i}\left(R\left(x_{k-1}\right), R\left(u_{k-1}\right), R\left(v_{k-1}\right)\right), i=\overline{1, n_{1}}$

$x_{k}^{i}=f_{3}^{i}\left(B\left(x_{k-1}\right), B\left(u_{k-1}\right), B\left(v_{k-1}\right)\right), i=\overline{n_{1}+1, n}$

$y_{k}^{i}=g_{1}^{i}\left(B\left(x_{k}\right), B\left(u_{k}\right)\right)+g_{2}^{i}\left(R\left(x_{k}\right), B\left(u_{k}\right)\right), i=\overline{1, p_{1}}$

$y_{k}^{i}=g_{3}^{i}\left(R\left(x_{k}\right), R\left(u_{k}\right)\right), i=\overline{p_{1}+1, p}$

where:

$x \in X=\mathbf{R}^{n_{1}} \times \mathbf{B}_{2}^{n_{2}}, n_{1}+n_{2}=n$ represent the set of the states of the system,

$u \in U=\mathbf{R}^{m_{1}} \times \mathbf{B}_{2}^{m_{2}}, m_{1}+m_{2}=n$ represent the set of the input signals of the system,

$v \in V=\mathbf{R}^{r_{1}} \times \mathbf{B}_{2}^{r_{2}}, r_{1}+r_{2}=r$ represent the set of the perturbations of the system,

$y \in Y=\mathbf{R}^{p_{1}} \times \mathbf{B}_{2}^{p_{2}}, p_{1}+p_{2}=p$ represent the set of the output signs of the system,

$f_{1}^{i}, i=\overline{1, n_{1}}$ are pseudo-boolean functions,

$f_{2}^{i}, i=\overline{1, n_{1}}$ are linear functions,

$f_{3}^{i}, i=\overline{n_{1} 1, n}$ are boolean functions,

$g_{1}^{i}, i=\overline{1, p_{1}}$ are pseudo-boolean functions,

$g_{2}^{i}, i=\overline{1, p_{1}}$ are linear functions,

$g_{3}^{i}, i=\overline{p_{1}+1, p}$ are boolean functions.

\section{Remarks:}

The signs of the quasi-boolean system are vectors with real numbers and boolean values elements. The complexity of the system is described through linear, pseudo-boolean and boolean functions. The informational state of the system is described using real numbers and truth values. Any boolean function can be expressed using a pseudo-boolean function. In order to model the learning (instructional) process, there will be used integer numbers from an interval, instead of real numbers, so the instructional system will become a finite states system. Using the interpolation formula for pseudo-boolean functions [10], the equations of the system become:

$$
\begin{aligned}
& x_{i}^{k}=\sum c_{\gamma} x_{k-1}^{\alpha_{n_{1}+1}}, \ldots, x_{k-1}^{\alpha_{n}}, u_{k-1}^{\alpha_{m_{1}+1}}, \ldots, u_{k-1}^{\alpha_{m}}, v_{k-1}^{\alpha_{r_{1}+1}}, \ldots, v_{k-1}^{\alpha_{r}}+f_{2}^{i}\left(R\left(x_{k-1}\right), R\left(u_{k-1}\right), R\left(v_{k-1}\right),\right. \\
& i=\overline{1, n}, c_{\gamma}=f_{1}^{i}\left(\alpha_{n_{1}+1}, \ldots, \alpha_{n}, \alpha_{m_{1}+1}, \ldots, \alpha_{m}, \alpha_{r_{1}+1}, \ldots, \alpha_{r}\right) \\
& y_{i}^{k}=\sum b_{\delta} x_{k-1}^{\delta_{n_{1}+1}}, \ldots, x_{k-1}^{\delta_{n}}, u_{k-1}^{\delta_{m_{1}+1}}, \ldots, u_{k-1}^{\delta_{m}}+g_{2}^{i}\left(R\left(x_{k-1}\right), R\left(u_{k-1}\right)\right), \\
& i=\overline{1, n}, b_{\delta}=g_{1}^{i}\left(\delta_{n_{1}+1}, \ldots, \delta_{n}, \delta_{m_{1}+1}, \ldots, \delta_{m}\right)
\end{aligned}
$$

The equations of the system can be expressed using linear polynomial dependent on the states, the perturbations and the inputs of the system. If the pseudo-boolean functions are linear pseudo-boolean functions, they can be expressed as follows:

$T(x)=c_{1} x_{1}+c_{2} x_{2}+\ldots+c_{n} x_{n}+d$, where

$c_{1}, c_{2}, \ldots, c_{n}, d$ are coefficients (in the system described in the paper, the coefficients are integer number).

The topic of the paper is not to describe the quasi-boolean systems. These quasi-boolean of systems (in particular cases) are observable, controllable and there may be defined regulation laws (feedback regulation and predictive regulation). The methods of solving linear pseudo-boolean equations could be found in [10]. 
Example:

One considers an online course with three modules. The initial state is:

$x_{0}=(0,0,0)$ i.e. there is no teaching

The inputs (commands) of the system are:

$u_{0}=(0,0,0)$ i.e. there is no teaching process

$u_{1}=(1,0,0)$ i.e. the system teaches module no. 1

$u_{2}=(0,1,0)$ i.e. the system teaches module no. 2

$u_{3}=(0,0,1)$ i.e. the system teaches module no. 3

The perturbations are:

$(0,0)$ i.e. the electrical power is interrupted

$(0,1)$ i.e. the Internet connection failed

$(1,0)$ i.e. the computer is failed

The equation of the system is:

$x_{k+1}=\left(x_{k}^{1} v_{k}^{1} v_{k}^{2}+u_{k}^{1} v_{k}^{1} v_{k}^{2}-x_{k}^{1} u_{k}^{1} v_{k}^{1} v_{k}^{2}, x_{k}^{2} v_{k}^{1} v_{k}^{2}+u_{k}^{2} v_{k}^{1} v_{k}^{2}-x_{k}^{2} u_{k}^{2} v_{k}^{1} v_{k}^{2}, x_{k}^{3} v_{k}^{1} v_{k}^{2}+u_{k}^{3} v_{k}^{1} v_{k}^{2}-x_{k}^{3} u_{k}^{3} v_{k}^{1} v_{k}^{2}\right)$

The reference value is: $y_{\text {ref }}^{*}=(1,1,1)$

If there is no perturbation, the evolution of the system is: $x_{1}=(0,0,0) x_{2}=(1,0,0) x_{3}=(1,1,0)$ $x(4)=(1,1,1)$

The regulator verifies the obtained value $(1,1,1)$ with the reference value $(1,1,1)$. If there is a difference between them (considering a perturbation), the output value could be $(0,1,1)$.

The error formula is:

$$
\begin{aligned}
& y_{\text {ref }}^{*}=\left(y_{\text {ref }}^{1}, y_{\text {ref }}^{2}, y_{\text {ref }}^{3}\right) \\
& y^{*}=\left(y^{* 1}, y^{* 2}, y^{* 3}\right) \\
& e=\left(y_{\text {ref }}^{1} \overline{y^{* 1}}, y_{\text {ref }}^{2} \overline{y^{* 2}}, y_{\text {ref }}^{3} \overline{y^{* 3}}\right)
\end{aligned}
$$

The patterns' analyzer described in section 2 is expressed as a boolean equation.

In the former case, the error is $e=(0,0,0)$ and the process finished with success. In the latter case, the error is $e=(1,0,0)$ and the feedback regulation has to order to teach the first module.

\section{An Application Example}

The objective of the learning(instructional) process:

At the end of the teaching session, students will be able to design a simple web page using HTML language and the web page will be written correctly. In order to achieve this goal, the system have to teach: (1) module no. 1: the structure of the web pages, (2) module no.2: the $\langle$ tml $\rangle,\langle$ head $\rangle$, $\langle$ title $\rangle$, $\langle$ body $\rangle,\langle p\rangle$ tags, (3) module no. 3: applications. For each module, the learning (instructional) system has a set of pedagogical resources.

The reference value is: $y_{\text {ref }}^{*}=(1,1,1,10)$, where

1 is the true value=the student has written a web page,

1 is the true value=the student has used the HTML language,

1 is the true value=the structure of the web page is correct

The perturbation considered are defined by the learning styles (visual, auditive, kinesthetic) and the age categories (three categories).

$(1,0,0,1,0,0)$ i.e. learning style $=$ visual and age category $=1$

$(0,1,0,1,0,0)$ i.e. learning style $=$ auditive and age category $=1$

$(0,0,1,1,0,0)$ i.e. learning style=kinesthetic and age category $=1$

$(1,0,0,0,1,0)$ i.e. learning style $=$ visual and age category $=2$

$(0,1,0,0,1,0)$ i.e. learning style $=$ auditive and age category $=2$

$(0,0,1,0,1,0)$ i.e. learning style $=$ kinesthetic and age category $=2$

$(1,0,0,0,0,1)$ i.e. learning style $=$ visual and age category $=3$ 
$(0,1,0,0,0,1)$ i.e. learning style $=$ auditive and age category $=3$

$(0,0,1,0,0,1)$ i.e. earning style=kinesthetic and age category $=3$

The instructional system has to teach three modules; for each module there is a set of pedagogical resources (noted with $P R_{k}=$ pedagogical resources with identification $k$ ).

The commands of the system are:

$(0,0,0,0)$ i.e. invoke a virtual meeting between the teacher and the student

$\left(1,0,0, P R_{1}\right)$ i.e. teach module no. 1 using the pedagogical resource with identification 1

$\left(1,0,0, P R_{2}\right)$ i.e. teach module no. 1 using the pedagogical resource with identification 2

$\left(1,0,0, P R_{3}\right)$ i.e. teach module no. 1 using the pedagogical resource with identification 3

$\left(1,0,0, P R_{4}\right)$ i.e. teach module no. 1 using the pedagogical resource with identification 4

$\left(1,0,0, P R_{5}\right)$ i.e. teach module no. 1 using the pedagogical resource with identification 5

$\left(0,1,0, P R_{6}\right)$ i.e. teach module no. 2 using the pedagogical resource with identification 6

$\left(0,1,0, P R_{7}\right)$ i.e. teach module no. 2 using the pedagogical resource with identification 7

$\left(0,1,0, P R_{8}\right)$ i.e. teach module no. 2 using the pedagogical resource with identification 8

$\left(0,0,1, P R_{9}\right)$ i.e. teach module no. 3 using the pedagogical resource with identification 9

$\left(0,0,1, P R_{10}\right)$ i.e. teach module no. 3 using the pedagogical resource with identification 10

$\left(0,0,1, P R_{11}\right)$ i.e. teach the module no. 3 using the pedagogical resource with identification 11

$\left(0,0,1, P R_{12}\right)$ i.e. teach module no. 3 using the pedagogical resource with identification 12

So, there are five pedagogical resources for module no. 1, three pedagogical resources for module no. 2, and four pedagogical resources for module no. 3 .

The equations of the system are:

$x_{k}^{1}=x_{k-1}^{1}+u_{k-1}^{1}-x_{k-1}^{1} u_{k-1}^{1}$

$x_{k}^{2}=x_{k-1}^{2}+u_{k-1}^{2}-x_{k-1}^{2} u_{k-1}^{2}$

$x_{k}^{3}=x_{k-1}^{3}+u_{k-1}^{3}-x_{k-1}^{3} u_{k-1}^{3}$

$x_{k}^{4}=x_{k-1}^{4}+u_{k-1}^{1} u_{k-1}^{4}$

$x_{k}^{5}=x_{k-1}^{5}+u_{k-1}^{2} u_{k-1}^{5}$

$x_{k}^{6}=x_{k-1}^{6}+u_{k-1}^{3} u_{k-1}^{6}$

A state of the form $(1,1,0,4,7,0)$ means that the student has followed the modules no.1 and 2 using the pedagogical resources no. 4 and 7.

The output is: $y=\left(x_{k}^{1} x_{k}^{2} x_{k}^{3}, x_{k}^{1} x_{k}^{2} x_{k}^{3}, x_{k}^{1} x_{k}^{2} x_{k}^{3}, \alpha x_{k}^{1} x_{k}^{2} x_{k}^{3}+\beta x_{k}^{1} x_{k}^{2} x_{k}^{3}+\gamma x_{k}^{1} x_{k}^{2} x_{k}^{3}\right)$, where $\alpha+\beta+\gamma=10$ are given coefficients.

This means that a student may obtain at the evaluation the grade $=10$, if the behaviour has the value 1 , the conditions have the value 1 and the criteria have the value 1 .

A scenario of the system's behavior is: a new student has to learn web pages. The student has a learning style and an age category. The predictive controller has learned which pedagogical resources fit with the profile of the student. This can be realized using AI learning techniques [13] or the algorithm proposed in [18].

The error is:

$e=y_{\text {ref }}^{4 *}-y^{4 *}+\alpha y_{\text {ref }}^{1 *} \overline{y^{1 *}}+\beta y_{\text {ref }}^{2 *} \overline{y^{2 *}}+\gamma y_{\text {ref }}^{3 *} \overline{y^{3 *}}$

If the error is too big, the feedback controller provides a command to invoke a virtual meeting between the teacher and the student.

\section{Problems of the Online Instructional Systems}

The major problems connected to the online instructional systems are posed by their complexity and uncertainty. Generally speaking, the uncertainty refers to the impossibility of exact prediction. The uncertainty is a feature of the human behaviour. The human factor is a part of the online instructional systems and the instructional process act on humans (figure no. 9). So, an online instructional system has 
to be viewed from the point of its complexity and uncertainty. The uncertainty of the system means that the inputs of the system don't determine exactly the outputs of the system. The outputs of the system have

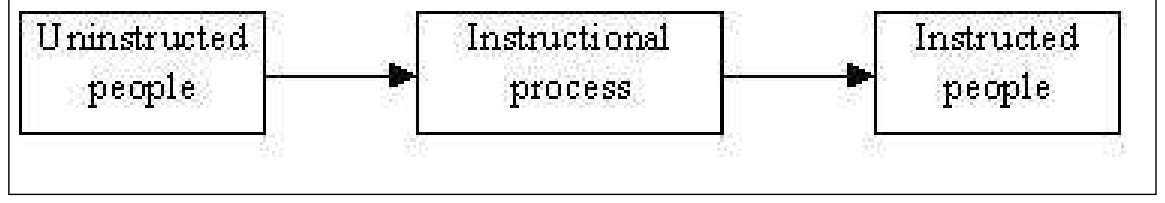

Figure 9: Inputs and outputs of the instructional process

to satisfy the standards knowledge according to the objectives of the instructional process. The authors of the paper "Defining Uncertainty a Conceptual Basis for Uncertainty Management in Model-Based Decision Support" [19] define the uncertainty concept taking into consideration three dimensions:

- the location of the uncertainty;

- the level of uncertainty;

- the nature of the uncertainty - the source of uncertainty, the imperfection of the knowledge, the diversity of the values, etc.

At a macro level, there may be distinguished two sources of the uncertainty:

1. the variability;

2. the limit of the knowledge.

The instructional systems are developing from the new scientific perspective of the analysis of their uncertainty. If we consider an instructional system with perturbation factors having null values, the outputs of the system cannot be predicted with accuracy. The instructional process is unpredictable and can not be reduced at linear sequences to produce predictable outputs. Considering the perturbation factors and the complexity of the relations between the parts of the system and the relations between system and the environment, the instructional system is a complex system. To obtain a reference value, the instructional system has to be defined as a complex adaptive system.

A Complex Adaptive System - (CAS) is a system with a lot of internal and external relations; the system can adapt itself and evaluate according to changes from the environment. It is important to see that there is not a precise boundary between the system and the environment. The system influences and changes the environment. An instructional system is linked with other systems, so that the changes have to be considered in terms of a co-evolution with other systems. An instructional system has to be designed from the viewpoint of the internal variable side and external variable side. The system records information about the environment, this information determines the behaviour of the system, the system transmits information to the environment and acts in accordance with the environment.

Another viewpoint of instructional process is the hermeneutic aspect, in which the learning is defined as an interpretation act, inseparable from the cultural and historical context. Jonassen [12] states that the instructional design theory has to consider the learning as an open system, which receives inputs from many sources, such as individual differences, emotional states, social and economical factors, demographical factors and so forth. The instructional designers have to understand the complex and holistic nature of the learning process and have not to isolate this kind of systems in closed systems.

\section{Summary and Conclusions}

In the perspective of the evolution of the whole society, it is necessary to approach the instructional process from the viewpoint of the systems theory. This perspective enables to solve the problems of 
the instructional process, in special considering the distance learning process. Practically, the distance instructional process, speaking about online learning process, can be successfully applied only using the techniques from systems theory and artificial intelligence. In this paper, the author has modeled the learning (instructional) process using the general systems theory. In this paper, the term of "quasiboolean system" was introduced in order to provide a mathematical model describing the behaviour of the instructional system. The AI techniques are used in the regulation of the system. The possible applications of the proposed approach may be found in the instructional system to teach Mathematics, Computer Science, Architecture [21], Physics, Chemistry, etc. This kind of approach enables solving the problems of the complexity and uncertainty of the instructional systems, especially those of the online instructional systems.

\section{Bibliography}

[1] B. H. Banathy, A Systems View of Education: Concepts and Principles for Effective Practice. Englewood Cliffs, N.J.: Educational Technology Publications, 1992.

[2] B. H. Banathy, Systems Inquiry and Its Application in Education, In D. H. Jonassen (ed), Handbook of research for educational communication and technology, New York: MacMillan Library Reference, 1996.

[3] D. Chen and W. Stroup, Toward a Conceptual Framework for Science and Technology Education for All, Journal of Science Education and Technology, Volume 2, No.3, 1993.

[4] M. Cole., P. Griffin, Contextual Factors in Education. Information Analyses Report. Prepared for Committee on Research in Mathematics Science, and Technology Education, Commission on Behavioral and Social Sciences and Education, National Research Council. Wisconsin Center for Education Research, Madison, 1987.

[5] W. Dick, L. Carey, The Systematic Design of Instruction, Glenview,IL.:Scott,Foresman, 1978.

[6] R.R. Du Mont, Distance Learning: A Systems View an Assessment and Review of the Literature, Research report, grant awarded by the Ohio Learning Network in cooperation with Research Center for Educational Technology Kent State University, 2002.

[7] J.D. Finn, Automation and Education: I. General aspects, AV Communication Review, 5(1), 1957.

[8] R. Gagné, L. Briggs, W.W. Wager,Principles of Instructional Design,(3rd ed.). New York, NY: Holt, Rinehart and Winston, Inc., 1988.

[9] G. Gonzalez, A Systematic Approach to Active and Cooperative Learning in CS1 and its Effects on CS2, Technical Symposium on Computer Science Education, Proceedings of the 37th SIGCSE technical symposium on Computer science education, 2006.

[10] P.L. Hammer(Ivanescu), S. Rudeanu, Methodes Booleennes en Recherche Operationelle, Dunod, Paris, 1970.

[11] C. Ilas, Teoria Sistemelor de Reglare Automata, Matrix Rom, Bucuresti, 2006.

[12] D.H. Jonassen, et al. Certainty, Determinism, and Predictability in Theories of Instructional Design: Lessons from Science, Educational Technology, (37)1, 1997.

[13] G. Moise, A Software System for Online Learning Applied in the Field of Computer Science, IJCCC, Volume: II, No: 1, 2007. 
[14] G. Moise, A rules Based on Context Methodology to Build the Pedagogical Resources, Proceedings of the 2nd International Conference on Virtual Learning, Bucharest University Press, 2007.

[15] M.G. Moore and G. Kearsley, Distance Education: A Systems View, Belmont, CA:Wadsworth Publishing Co., 1996.

[16] M.G. Moore,Theory of Transactional Distance, In D. Keegan (Ed.) Theoretical Principles of Distance Education. New York: Routledge, 1993.

[17] F. Saba, R.L. Shearer,Verifying Key Theoretical concepts in a Dynamic Model of Distance Education, The American Journal of Distance Education, 8 (1), 1994.

[18] A.D. Styliadis, I. D. Karamitsos, D. I. Zachariou, Personalized e-Learning Implementation - The GIS Case, International Journal of Computers, Communications \& Control, Vol. I, No.1, 2006.

[19] W.E. Walker, P. Harremöees, J. Rotmans, J.P. VAN DER Sluijs, M.B.A. VAN Asselt, P. Janssen AND M.P. Krayer VON Krauss, Defining Uncertainty A Conceptual Basis for Uncertainty Management in Model-Based Decision Support, Integrated Assessment, Vol. 4, No. 1, 2003.

[20] N. Wiener, The Human Use of Human Beings : cybernetics and society, Second Edition Revised, Doubleday Anchor, 1954.

[21] A.D. Styliadis, E-learning Documentation of Historical Living Systems with 3D Modeling Functionality, Informatica, Vol. 18, No. 3, pp. 419-446, 2007.

\author{
Gabriela Moise \\ Petroleum-Gas University of Ploiesti \\ Computer Science Department \\ no. 39 Bd. Bucuresti, Ploiesti, Romania \\ E-mail: gmoise@upg-ploiesti.ro \\ Received: February 13, 2007
}

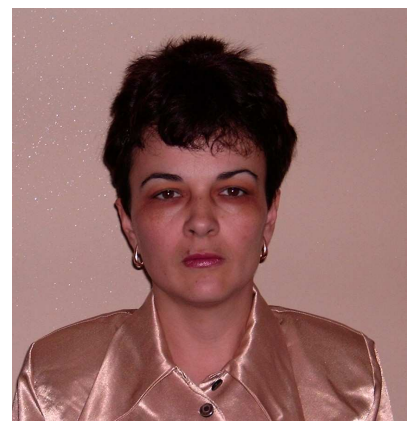

Gabriela Moise (born on February 13, 1969) graduated the Faculty of Mathematics, specialization Informatics of the Bucharest University. She worked in the software industry, developing IT solutions. Since 2003 she is Lecturer at Petroleum-Gas University of Ploiesti. Her research fields are: e-learning, graph theory, pedagogical agents, knowledge representation, e-health. She has (co)authored seven books and more than twenty research papers. She has participated to many international conferences in the elearning and e-business area. 